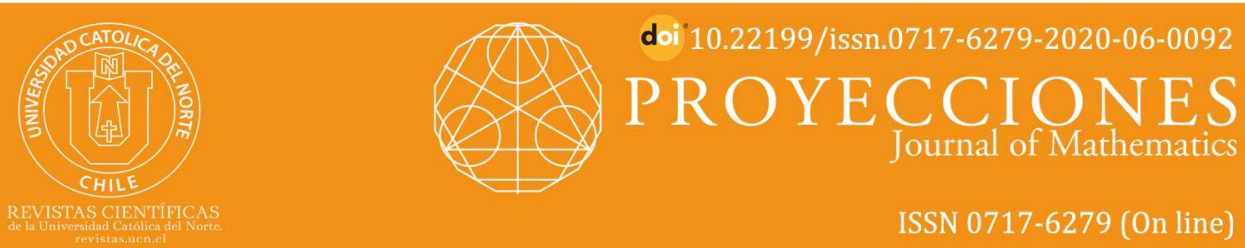

\title{
On generalizations of graded second submodules
}

\author{
Mashhoor Refai ${ }^{1}$ (1) orcid.org/0000-0001-7436-5385 \\ Rashid Abu-Dawwas ${ }^{2}$ (0) orcid.org/0000-0001-8998-7590 \\ ${ }^{1}$ President of Princess Sumaya University for Technology, Jordan. \\ 『m.refai@psut.edu.jo \\ ${ }^{2}$ Yarmouk University, Dept. of Mathematics, Jordan. \\ ⿶rrashid@yu.edu.jo
}

Received: February 2019 | Accepted: April 2020

\section{Abstract:}

Let $G$ be a group with identity e, $R$ be a commutative $G$-graded ring with unity 1 and $M$ be a $G$-graded $R$-module. In this article, we introduce and study two generalizations of graded second submodules, namely, graded 2-absorbing second submodules and graded strongly 2- absorbing second submodules. Also, we introduce and study the concept of graded quasi 2 -absorbing second submodules, that is a generalization for graded strongly 2-absorbing second submodules.

Keywords: Graded second submodules; Graded 2-absorbing submodules; Graded strongly 2-absorbing submodules; Graded 2-absorbing second submodules; Graded strongly 2absorbing second submodules; Graded quasi 2-absorbing second submodules.

MSC (2020): 13A02, 16W50.

\section{Cite this article as (IEEE citation style):}

M. Refai and R. Abu-Dawwas, "On generalizations of graded second submodules", Proyecciones (Antofagasta, On line), vol. 39, no. 6, pp. 1537-1554, Dec. 2020, doi: 10.22199/issn.07176279-2020-06-0092.

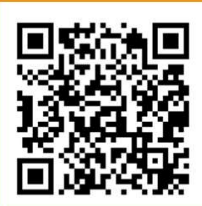

Article copyright: (C) 2020 Mashhoor Refai and Rashid Abu-Dawwas. This is an open access article distributed under the terms of the Creative Commons License, which permits unrestricted use and distribution provided the original author and source are credited. 


\section{Introduction}

Throughout this article, $G$ will be a group with identity $e$ and $R$ will be a commutative ring with a nonzero unity $1 . R$ is said to be $G$-graded if $R=\bigoplus_{g \in G} R_{g}$ with $R_{g} R_{h} \subseteq R_{g h}$ for all $g, h \in G$ where $R_{g}$ is an additive subgroup of $R$ for all $g \in G$. The elements of $R_{g}$ are called homogeneous of degree $g$. Consider $\operatorname{supp}(R, G)=\left\{g \in G: R_{g}=0\right\}$. If $x \in R$, then $x$ can be written as $\sum_{g \in G} x_{g}$, where $x_{g}$ is the component of $x$ in $R_{g}$. Also, $h(R)=\bigcup_{g \in G} R_{g}$. Moreover, it has been proved in [21] tha $R_{e}$ is a subring of $R$ and $1 \in R_{e}$.

Let $I$ be an ideal of a graded ring $R$. Then $I$ is said to be graded ideal if $I=\bigoplus_{g \in G}\left(I \cap R_{g}\right)$, i.e., for $x \in I, x=\sum_{g \in G} x_{g}$ where $x_{g} \in I$ for all $g \in G$. Let $R$ be a $G$-graded ring and $I$ be a graded ideal of $R$. Then $R / I$ is $G$-graded by $(R / I)_{g}=\left(R_{g}+I\right) / I$ for all $g \in G$.

Assume that $M$ is a left $R$-module. Then $M$ is said to be $G$-graded if $M=\bigoplus_{g \in G} M_{g}$ with $R_{g} M_{h} \subseteq M_{g h}$ for all $g, h \in G$ where $M_{g}$ is an additive subgroup of $M$ for all $g \in G$. The elements of $M_{g}$ are called homogeneous of degree $g$. Also, we consider $\operatorname{supp}(M, G)=\left\{g \in G: M_{g}=0\right\}$. It is clear that $M_{g}$ is an $R_{e}$-submodule of $M$ for all $g \in G$. Moreover, $h(M)=\bigcup_{g \in G} M_{g}$. Let $N$ be an $R$-submodule of a graded $R$-module $M$. Then $N$ is said to be graded $R$-submodule if $N=\bigoplus_{g \in G}\left(N \cap M_{g}\right)$, i.e., for $x \in N, x=\sum_{g \in G} x_{g}$ where $x_{g} \in N$ for all $g \in G$. Let $M$ be a $G$-graded $R$-module and $N$ be a graded $R$-submodule of $M$. Then $M / N$ is a graded $R$-module by $(M / N)_{g}=\left(M_{g}+N\right) / N$ for all $g \in G$.

Lemma 1.1. ([16]) Let $R$ be a $G$-graded ring and $M$ be a $G$-graded $R$ module.

1. If $I$ and $J$ are graded ideals of $R$, then $I+J$ and $I \cap J$ are graded ideals of $R$.

2. If $N$ and $K$ are graded $R$-submodules of $M$, then $N+K$ and $N \cap K$ are graded $R$-submodules of $M$. 
3. If $N$ is a graded $R$-submodule of $M, r \in h(R), x \in h(M)$ and $I$ is a graded ideal of $R$, then $R x, I N$ and $r N$ are graded $R$-submodules of $M$. Moreover, $\left(N:_{R} M\right)=\{r \in R: r M \subseteq N\}$ is a graded ideal of $R$.

Also, it has been proved in [17] that if $N$ is a graded $R$-submodule of $M$, then $\operatorname{Ann}_{R}(N)=\{r \in R: r N=\{0\}\}$ is a graded ideal of $R$.

Graded prime submodules have been introduced by Atani in [12]. A proper graded $R$-submodule $N$ of $M$ is said to be graded prime if whenever $r \in h(R)$ and $m \in h(M)$ such that $r m \in N$, then either $m \in N$ or $r \in\left(N:_{R} M\right)$. Graded prime submodules have been widely studied by several authors, for more details one can look in [1], [2], [4] and [8].

Let $M$ and $S$ be two $G$-graded $R$-modules. An $R$-homomorphism $f$ : $M \rightarrow S$ is said to be graded $R$-homomorphism if $f\left(M_{g}\right) \subseteq S_{g}$ for all $g \in G$ (see [21]). Graded second submodules have been introduced by AnsariToroghy and Farshadifar in [9]. A nonzero graded $R$-submodule $N$ of $M$ is said to be graded second if for each $a \in h(R)$, the graded $R$-homomorphism $f: N \rightarrow N$ defined by $f(x)=a x$ is either surjective or zero. In this case, $A n n_{R}(N)$ is a graded prime ideal of $R$. Graded second submodules have been wonderfully studied by Çeken and Alkan in [14]. On the other hand, graded secondary modules have been introduced by Atani and Farzalipour in [13]. A nonzero graded $R$-module $M$ is said to be graded secondary if for each $a \in h(R)$, the graded $R$-homomorphism $f: M \rightarrow M$ defined by $f(x)=a x$ is either surjective or nilpotent.

In [20], Naghani and Moghimi gave a generalization of graded prime ideals, called graded 2-absorbing ideals. A proper graded ideal $P$ of $R$ is said to be graded 2-absorbing if whenever $a, b, c \in h(R)$ such that $a b c \in P$, then either $a b \in P$ or $a c \in P$ or $b c \in P$. Graded 2-absorbing ideals have been admirably studied in [6].

The authors in [5] extended graded 2-absorbing ideals to graded 2absorbing submodules. A proper graded $R$-submodule $N$ of $M$ is said to be graded 2-absorbing if whenever $a, b \in h(R)$ and $m \in h(M)$ such that $a b m \in N$, then either $a m \in N$ or $b m \in N$ or $a b \in\left(N:_{R} M\right)$. Graded 2-absorbing submodules have been deeply studied in [7].

In [15], a proper $\mathbf{Z}$-graded $R$-submodule $N$ of $M$ is said to be graded completely irreducible if whenever $N=\bigcap_{k \in \Delta} N_{k}$ where $\left\{N_{k}\right\}_{k \in \Delta}$ is a family of $\mathbf{Z}$-graded $R$-submodules of $M$, then $N=N_{k}$ for some $k \in \Delta$. In 
[19], the concept of graded completely irreducible submodules has been extended into $G$-graded case, for any group $G$. It has been proved that every graded $R$-submodule of $M$ is an intersection of graded completely irreducible $R$-submodules of $M$. In many instances, we use the following basic fact without further discussion.

Remark 1.2. Let $N$ and $L$ be two graded $R$-submodules of $M$. To prove that $N \subseteq L$, it is enough to prove that: If $K$ is a graded completely irreducible $R$-submodule of $M$ such that $L \subseteq K$, then $N \subseteq K$.

The purpose of our article is to follow [11] in order to introduce and study the concept of graded 2-absorbing second submodules, that is a generalization of graded second submodules. A nonzero graded $R$-submodule $N$ of $M$ is said to be graded 2-absorbing second if whenever $x, y \in h(R)$ and $K$ is a graded completely irreducible $R$-submodule of $M$ such that $x y N \subseteq K$, then either $x N \subseteq K$ or $y N \subseteq K$ or $x y \in A n n_{R}(N)$. Also, we follow [11] to introduce another generalization, namely, graded strongly 2-absorbing second submodules. A nonzero graded $R$-submodule $N$ of $M$ is said to be graded strongly 2-absorbing second if whenever $x, y \in h(R)$ and $K$ is a graded $R$-submodule of $M$ such that $x y N \subseteq K$, then either $x N \subseteq K$ or $y N \subseteq K$ or $x y \in A n n_{R}(N)$.

In Corollary 3.4, we prove that if $N$ is a graded strongly 2-absorbing second $R$-submodule of $M$, then $A n n_{R}(N)$ is a graded 2-absorbing ideal of $R$, and in Example 3.5, we show that the converse is not true in general. Motivated by this, we introduce and study a generalization for graded strongly 2-absorbing second submodules. A nonzero graded $R$-submodule $N$ of $M$ is said to be graded quasi 2-absorbing second if $\operatorname{Ann}(N)$ is a graded 2 -absorbing ideal of $R$. Related results have been obtained.

\section{Graded 2-Absorbing Second Submodules}

In this section, we introduce and study the concept of graded 2-absorbing second submodules.

Definition 2.1. Let $M$ be a graded $R$-module and $N$ be a nonzero graded $R$-submodule of $M$. Then $N$ is said to be a graded 2-absorbing second $R$-submodule of $M$ if whenever $x, y \in h(R)$ and $K$ is a graded completely irreducible $R$-submodule of $M$ such that $x y N \subseteq K$, then either $x N \subseteq K$ or $y N \subseteq K$ or $x y \in A n n_{R}(N)$. 
Example 2.2. Let $R=\mathbf{Z}, M=\mathbf{Z}_{n}[i]$ and $G=\mathbf{Z}_{4}$. Then $R$ is $G$-graded by $R_{0}=\mathbf{Z}$ and $R_{1}=R_{2}=R_{3}=\{0\}$. Also, $M$ is $G$-graded by $M_{0}=\mathbf{Z}_{n}$, $M_{2}=i \mathbf{Z}_{n}$ and $M_{1}=M_{3}=\{0\}$. Consider the graded $R$-submodule $N=\mathbf{Z}_{n}$ of $M$. If $n=p$ or $n=p q$ where $p, q$ are primes, then $N$ is a graded 2absorbing second $R$-submodule of $M$.

Example 2.3. Let $R=\mathbf{Z}, M=\mathbf{Z}[i]$ and $G=\mathbf{Z}_{2}$. Then $R$ is $G$-graded by $R_{0}=\mathbf{Z}$ and $R_{1}=\{0\}$. Also, $M$ is $G$-graded by $M_{0}=\mathbf{Z}$ and $M_{1}=$ $i \mathbf{Z}$. Consider the graded $R$-submodule $N=n \mathbf{Z}$ of $M$. Obviously, $n=$ $p_{1}^{r_{1}} p_{2}^{r_{2}} \ldots . . . p_{k}^{r_{k}}$ where $p_{i}^{r_{i}}(1 \leq i \leq k)$ are distinct primes. Now, $p_{1} \in h(R)$ and $K=p_{1}^{r_{1}+2} \mathbf{Z}$ is a graded completely irreducible $R$-submodule of $M$ such that $p_{1} p_{1} N \subseteq K$. But $p_{1} N \subseteq K$ and $p_{1} p_{1} \notin A n n_{R}(N)=\{0\}$. Hence, $N$ is not graded 2-absorbing second $R$-submodule of $M$.

Remark 2.4. Consider the $\mathbf{Z}$-module $\mathbf{Z}$ and assume it is $G$-graded by any group $G$. Since the only graded submodules are $n \mathbf{Z}$, then by Example 2.3, $\mathbf{Z}$ has no graded 2-absorbing second submodules.

Let $\Omega(M)$ be the set of all graded completely irreducible $R$-submodules of $M$. Assume that $P$ is a graded prime ideal of $R$ and $N$ is a graded $R$-submodule of $M$. Then we define

$$
I_{P}^{M}(N)=\bigcap_{K \in \Omega(M)}\{K: r N \subseteq K \text { for some } r \in h(R)-P\}
$$

. The following lemma gives some characterizations for graded second $R$ submodules.

Lemma 2.5. Let $N$ be a graded $R$-submodule of a graded $R$-module $M$. Then the following are equivalent.

1. If $N=\{0\}, K$ is a graded completely irreducible $R$-submodule of $M$ and $r \in h(R)$ such that $r N \subseteq K$, then either $r N=\{0\}$ or $N \subseteq K$.

2. $N$ is a graded second $R$-submodule of $M$.

3. $P=A n n_{R}(N)$ is a graded prime ideal of $R$ and $I_{P}^{M}(N)=N$.

Proof. $\quad(1) \Rightarrow(2)$ : Suppose that $r \in h(R)$ and $r N=\{0\}$. If $r N \subseteq K$ for some graded completely irreducible $R$-submodule $K$ of $M$, then by assumption, $N \subseteq K$. Hence, $N \subseteq r N$. (2) $\Rightarrow(3)$ : By [9], $P=A n n_{R}(N)$ is a graded prime ideal of $R$. Now,let $K$ be a graded completely irreducible 
$R$-submodule of $M$ and $r \in h(R)-P$ such that $r N \subseteq K$. Then $N \subseteq K$ by assumption. Therefore, $N \subseteq I_{P}^{M}(N)$. The reverse inclusion is clear. $(3) \Rightarrow(1)$ : Since $A n n_{R}(N)$ is a graded prime ideal of $R, N=\{0\}$. Let $K$ be a graded completely irreducible $R$-submodule of $M$ and $r \in h(R)$ such that $r N \subseteq K$. Suppose that $r N=\{0\}$. Then $r \in h(R)-P$. Therefore, $I_{P}^{M}(N) \subseteq K$. But $I_{P}^{M}(N)=N$ by assumption. Hence, $N \subseteq K$, as desired.

Proposition 2.6. Let $M$ be a graded $R$-module. If either $L$ is a graded second $R$-submodule of $M$ or $L$ is a sum of two graded second $R$-submodules of $M$, then $L$ is a graded 2-absorbing second $R$-submodule of $M$.

Proof. The first assertion is clear. Let $N$ and $L$ be two graded second $R$-submodules of $M$. We show that $N+L$ is a graded 2-absorbing second $R$ submodules of $M$. Let $x, y \in h(R)$ and $K$ is a graded completely irreducible $R$-submodule of $M$ such that $x y(N+L) \subseteq K$. Since $N$ is graded second, either $x y N=\{0\}$ or $N \subseteq K$ by Lemma 2.5. Similarly, either $x y L=\{0\}$ or $L \subseteq K$. If $x y N=x y L=\{0\}$, then we are done. Also, if $N \subseteq K$ and $L \subseteq K$, then we are done. Assume that $x y N=\{0\}$ and $L \subseteq K$. Then $x N=\{0\}$ or $y N=\{0\}$ because $A n n_{R}(N)$ is a graded prime ideal of $R$. If $x N=\{0\}$, then $x(N+L) \subseteq x N+L \subseteq L \subseteq K$. Similarly, if $y N=\{0\}$, we have $y(N+L) \subseteq K$ as desired.

Proposition 2.7. Let $M$ be a graded R-module. If $L$ is a graded secondary $R$-submodule of $M$ and $R / A n n_{R}(L)$ has no nonzero nilpotent homogeneous element, then $L$ is a graded 2 -absorbing second $R$-submodule of $M$.

Proof. Let $x, y \in h(R)$ and $K$ be a graded completely irreducible $R$ submodule of $M$ such that $x y L \subseteq K$. If $x L \subseteq K$ or $y L \subseteq K$, then we are done. Suppose that $x L \subseteq K$ and $y L \subseteq K$. Then $x, y \in R / A n n_{R}(L)$. Thus, $(x y)^{r} \in A n n_{R}(L)$ for some positive integer $r$. Therefore, $x y \in A n n_{R}(L)$ since $R / A n n_{R}(L)$ has no nonzero nilpotent homogeneous element. Hence, $L$ is a graded 2-absorbing second $R$-submodule of $M$.

Proposition 2.8. Let $M$ be a $G$-graded $R$-module, $I$ be a graded ideal of $R$ and $L$ be a graded 2-absorbing second $R$-submodule of $M$. If $x \in h(R)$ and $K$ is a graded completely irreducible $R$-submodule of $M$ such that $x I L \subseteq K$, then either $x L \subseteq K$ or $x I \subseteq A n n_{R}(L)$ or $I_{g} L \subseteq K$ for some $g \in G$. 
Proof. Suppose that $x L \subseteq K$ and $x I \subseteq A n n_{R}(L)$. Then there exists $y \in I$ such that $x y L=\{0\}$, and then there exists $g \in G$ such that $x y_{g} L=$ $\{0\}$ where $y_{g} \in I$ since $I$ is graded. Now, since $L$ is graded 2-absorbing second and $x y_{g} L \subseteq K$, we have $y_{g} L \subseteq K$. We show that $I_{g} L \subseteq K$. Let $z_{g} \in I_{g}$. Then $\left(y_{g}+z_{g}\right) x L \subseteq K$. Hence, either $\left(y_{g}+z_{g}\right) L \subseteq L$ or $\left(y_{g}+z_{g}\right) x \in A n n_{R}(L)$. If $\left(y_{g}+z_{g}\right) L \subseteq K$, then since $y_{g} L \subseteq K$, we have $z_{g} L \subseteq K$. If $\left(y_{g}+z_{g}\right) x \in A n n_{R}(L)$, then $z_{g} x \notin A n n_{R}(L)$, but $z_{g} x L \subseteq K$. Thus, $z_{g} L \subseteq K$. Hence, we conclude that $I_{g} L \subseteq K$.

Lemma 2.9. Let $M$ be a $G$-graded $R$-module and $N$ a graded $R$-submodule of $M$. If $r \in h(R)$, then $(N: M r)=\{m \in M: r m \in N\}$ is a graded $R$ submodule of $M$.

Proof. Clearly, $\left(N:_{M} r\right)$ is a graded $R$-submodule of $M$. Let $m \in$ $\left(N:_{M} r\right)$. Then $r m \in N$. Now, $m=\sum_{g \in G} m_{g}$ where $m_{g} \in M_{g}$ for all $g \in G$.

Since $r \in h(R), r \in R_{h}$ for some $h \in G$ and then $r m_{g} \in M_{h g} \subseteq h(M)$ for all $g \in G$ such that $\sum_{g \in G} r m_{g}=r\left(\sum_{g \in G} m_{g}\right)=r m \in N$. Since $N$ is graded, $r m_{g} \in N$ for all $g \in G$ which implies that $m_{g} \in\left(N:_{M} r\right)$ for all $g \in G$. Hence, $(N: M r)$ is a graded $R$-submodule of $M$.

In [23], a graded $R$-module $M$ is said to be graded cocyclic if the sum of all graded minimal $R$-submodules of $M$ is a large and graded simple $R$-submodule of $M$.

Lemma 2.10. A graded $R$-submodule $K$ of $M$ is a graded completely irreducible $R$-submodule of $M$ if and only if $M / K$ is a graded cocyclic $R$-module.

Proof. It follows from ([18], Remark 1.1).

Lemma 2.11. Let $K$ be a graded completely irreducible $R$-submodule of $M$. Then $\left(K:_{M} r\right)$ is a graded completely irreducible $R$-submodule of $M$ for all $r \in h(R)$.

Proof. This follows from Lemma 2.9, Lemma 2.10 and that $M /\left(K:_{M}\right.$ $r) \cong(r M+K) / K$.

Proposition 2.12. Let $L$ be a graded 2-absorbing second $R$-submodule of $M$ and $K$ is a graded completely irreducible $R$-submodule of $M$ such that $L \subseteq K$, Then $\left(K:_{R} L\right)$ is a graded 2-absorbing ideal of $R$. 
Proof. Since $L \subseteq K$, we have $\left(K:_{R} L\right)=R$. Let $x, y, z \in h(R)$ such that $x y z \in\left(K:_{R} L\right)$. Then $x y L \in\left(K:_{M} z\right)$. Thus $x L \subseteq\left(K:_{M} z\right)$ or $y L \subseteq\left(K:_{M} z\right)$ or $x y L=\{0\}$ since $L$ is graded 2-absorbing second and $\left(K:_{M} z\right)$ is a graded completely irreducible $R$-submodule of $M$ by Lemma 2.11. Therefore, $x z \in\left(K:_{R} L\right)$ or $y z \in\left(K:_{R} L\right)$ or $x y \in\left(K:_{R} L\right)$. Hence, $\left(K:_{R} L\right)$ is a graded 2-absorbing ideal of $R$.

Corollary 2.13. If $M$ is a graded cocyclic $R$-module and $L$ is a graded 2absorbing second $R$-submodule of $M$, then $A n n_{R}(L)$ is a graded 2-absorbing ideal of $R$.

Proof. Since $M$ is graded cocyclic, $\{0\}$ is a graded completely irreducible $R$-submodule of $M$ by Lemma 2.10. Thus the result follows from Proposition 2.12 .

Proposition 2.14. Let $L$ be a graded 2-absorbing second $R$-submodule of $M$. Then $x^{n} L=x^{n+1} L$ for all $x \in h(R)$ and $n \geq 2$.

Proof. It is enough to prove that $x^{2} L=x^{3} L$. Let $x \in h(R)$. Then clearly, $x^{3} L \subseteq x^{2} L$. Let $K$ be a graded completely irreducible $R$-submodule of $M$ such that $x^{3} L \subseteq K$. Then $x^{2} L \subseteq\left(K:_{M} x\right)$. Thus $x L \subseteq\left(K:_{M} x\right)$ or $x^{2} L=\{0\}$ since $L$ is graded 2-absorbing second submodule of $M$ and $\left(K:_{M} x\right)$ is a graded completely irreducible $R$-submodule of $M$ by Lemma 2.11. Therefore, $x^{2} L \subseteq K$. Hence, $x^{2} L=x^{3} L$.

Proposition 2.15. Let $L$ be a graded 2-absorbing second $R$-submodule of $M$. If $A n n_{R}(L)$ is a graded prime ideal of $R$, then $\left(K:_{R} L\right)$ is a graded prime ideal of $R$ for all graded completely irreducible $R$-submodule $K$ of $M$ with $L \subseteq K$.

Proof. Let $K$ be a graded completely irreducible $R$-submodule of $M$ such that $L \subseteq K$. Assume that $x, y \in h(R)$ such that $x y \in\left(K:_{R} L\right)$. Then $x y L \subseteq K$, and then $x L \subseteq K$ or $y L \subseteq K$ or $x y L=\{0\}$. If $x y L=\{0\}$, then $x y \in A n n_{R}(L)$, and then $x L=\{0\}$ or $y L=\{0\}$. So, in all cases, we have $x L \subseteq K$ or $y L \subseteq K$, which implies that $x \in\left(K:_{R} L\right)$ or $y \in\left(K:_{R} L\right)$. Hence, $\left(K:_{R} L\right)$ is a graded prime ideal of $R$.

Proposition 2.16. Let $L$ be a graded 2-absorbing second $R$-submodule of $M$. If $\operatorname{Grad}\left(\operatorname{Ann}_{R}(L)\right)=P$ for some graded prime ideal $P$ of $R$ and $K$ is a graded completely irreducible $R$-submodule of $M$ such that $L \subseteq K$, then $\operatorname{Grad}\left(\left(K:_{R} L\right)\right)$ is a graded prime ideal of $R$ containing $P$. 
Proof. Let $x, y \in h(R)$ such that $x y \in G r a d\left(\left(K:_{R} L\right)\right)$. Then $x^{r} y^{r} L \subseteq$ $K$ for some positive integer $r$, and then $x^{r} L \subseteq K$ or $y^{r} L \subseteq K$ or $x^{r} y^{r} L=$ $\{0\}$. If $x^{r} L \subseteq K$ or $y^{r} L \subseteq K$, then we are done. Suppose that $x^{r} y^{r} L=$ $\{0\}$. Then $x y \in \operatorname{Grad}\left(\operatorname{Ann}_{R}(L)\right)=P$. Thus $x \in P$ or $y \in P$. Clearly, $P=\operatorname{Grad}\left(\operatorname{Ann}_{R}(L)\right) \subseteq \operatorname{Grad}\left(\left(K:_{R} L\right)\right)$. Therefore, $x \in \operatorname{Grad}\left(\left(K:_{R} L\right)\right)$ or $y \in \operatorname{Grad}\left(\left(K:_{R} L\right)\right)$.

\section{Graded Strongly 2-Absorbing Second Submodules}

In this section, we introduce and study the concept of graded strongly 2-absorbing second submodules.

Definition 3.1. Let $M$ be a graded $R$-module. Then a nonzero graded $R$-submodule $N$ of $M$ is said to be graded strongly 2-absorbing second if whenever $x, y \in h(R)$ and $K$ is a graded $R$-submodule of $M$ such that $x y N \subseteq K$, then either $x N \subseteq K$ or $y N \subseteq K$ or $x y \in \operatorname{Ann}_{R}(N)$.

Clearly, every graded strongly 2-absorbing second submodule is a graded 2-absorbing second submodule. This motivates the following question.

Question 3.2. Let $M$ be a graded R-module. Is every graded 2-absorbing second $R$-submodule of $M$ a graded strongly 2-absorbing second $R$-submodule of $M$ ?

Proposition 3.3. Let $N$ be a graded $R$-submodule of $M$. Then $N$ is a graded strongly 2-absorbing second $R$-submodule of $M$ if and only if for every $x, y \in h(R)$, we have $x y N=x N$ or $x y N=y N$ or $x y N=\{0\}$.

Proof. Suppose that $N$ is a graded strongly 2-absorbing second $R$ submodule of $M$. Then $N=\{0\}$. Let $x, y \in h(R)$. Then $x y N \subseteq x y N$, which implies that $x N \subseteq x y N$ or $y N \subseteq x y N$ or $x y N=\{0\}$. Thus $x y N=$ $x N$ or $x y N=y N$ or $x y N=\{0\}$. The converse is clear.

Corollary 3.4. If $N$ is a graded strongly 2-absorbing second $R$-submodule of $M$, then $A n n_{R}(N)$ is a graded 2-absorbing ideal of $R$.

Proof. Let $x, y, z \in h(R)$ such that $x y z \in A n n_{R}(N)$. Then by Proposition 3.3, we have $x y N=x N$ or $x y N=y N$ or $x y N=\{0\}$. If $x y N=\{0\}$, then we are done. Suppose that $x y N=x N$. Then $z x N \subseteq z x y N=\{0\}$. Similarly, if $x y N=y N$.

The following example shows that the converse of Corollary 3.4 is not true in general. 
Example 3.5. In Example 2.3, $N=\langle p\rangle$ (where $p$ is a prime number) is a graded $R$-submodule of $M=\mathbf{Z}[i]$ such that $A n n_{R}(N)=\{0\}$ is a graded 2-absorbing ideal of $R$, but $N$ is not a graded strongly 2-absorbing second $R$-submodule of $\mathbf{Z}[i]$.

Corollary 3.6. Let $N$ be a graded strongly 2-absorbing second $R$-submodule of $M$. If $L$ is a graded $R$-submodule of $M$ such that $N \subseteq L$, then $\left(L:_{R} N\right)$ is a graded 2-absorbing ideal of $R$.

Proof. Let $x, y, z \in h(R)$ such that $x y z \in\left(L:_{R} N\right)$. Then $x y z N \subseteq L$, and then $x z N \subseteq L$ or $y z N \subseteq L$ or $x y z N=\{0\}$. If $x z N \subseteq L$ or $y z N \subseteq L$, then we are done. If $x y z N=\{0\}$, then the result follows by Corollary 3.4.

A graded $R$-module $M$ is said to be graded comultiplication if for every graded $R$-submodule $N$ of $M$ there exists a graded ideal $J$ of $R$ such that $N=\left(0:_{M} J\right)$, equivalently, for every graded $R$-submodule $N$ of $M$, we have $N=\left(0:_{M} A n n_{R}(N)\right)$. The concept of graded comultiplication modules was introduced by H. Ansari-Toroghy and F. Farshadifar in [10]. Some generalizations on graded comultiplication modules have been introduced in [3]. The next proposition shows that the converse of Corollary 3.4 is true if $M$ is a graded comultiplication $R$-module.

Proposition 3.7. Let $M$ be a graded comultiplication $R$-module. If $L$ is a graded $R$-submodule of $M$ such that $A n n_{R}(L)$ is a graded 2-absorbing ideal of $R$, then $L$ is a graded strongly 2 -absorbing second $R$-submodule of $M$. In particular, $L$ is a graded 2-absorbing second $R$-submodule of $M$.

Proof. Let $x, y \in h(R)$ and $K$ be a graded $R$-submodule of $M$ such that $x y L \subseteq K$. Then $A n n_{R}(K) x y L=\{0\}$. So, $A n n_{R}(K) x L=\{0\}$ or $A n n_{R}(K) y L=\{0\}$ or $x y L=\{0\}$. If $x y L=\{0\}$, then we are done. If $A n n_{R}(K) x L=\{0\}$ or $A n n_{R}(K) y L=\{0\}$, then $A n n_{R}(K) \subseteq A n n_{R}(x L)$ or $A n n_{R}(K) \subseteq A n n_{R}(y L)$. Hence, $x L \subseteq K$ or $y L \subseteq K$ since $M$ is graded comultiplication.

Remark 3.8. Example 3.5 Shows that the condition that $M$ is a graded comultiplication $R$-module in Proposition 3.7 is necessary since $M$ is not a graded comultiplication $R$-module.

Corollary 3.9. Let $M$ be a graded comultiplication $R$-module. If $M$ is graded cocyclic, then every graded 2-absorbing second $R$-submodule of $M$ is graded strongly 2-absorbing second. 
Proof. Let $L$ be a graded 2-absorbing second $R$-submodule of $M$. Then by Corollary 2.13, $A n n_{R}(L)$ is a graded 2-absorbing ideal of $R$, and then the result follows by Proposition 3.7.

Proposition 3.10. Let $N$ be a graded strongly 2-absorbing second $R$ submodule of $M$. If $\left(K \cap L:_{R} N\right)$ is a graded prime ideal of $R$ for all graded completely irreducible $R$-submodules $K$ and $L$ of $M$ with $N \subseteq K \cap L$, then $A n n_{R}(N)$ is a graded prime ideal of $R$.

Proof. Suppose that $A n n_{R}(N)$ is not a graded prime ideal of $R$. Then there exist $x, y \in h(R)$ such that $x y N=\{0\}, x N=\{0\}$ and $y N=\{0\}$. So, there exist graded completely irreducible $R$-submodules $K$ and $L$ of $M$ such that $x N \subseteq K$ and $y N \subseteq L$. Now, $x y N \subseteq K \bigcap L$, which implies that $x y \in\left(K \cap L:_{R} N\right)$, and then $x N \subseteq K \bigcap L$ or $y N \subseteq K \bigcap L$. In both cases, we have a contradiction.

Lemma 3.11. Suppose that $f: M \rightarrow S$ is a graded $R$-homomorphism of graded $R$-modules.

1. If $f$ is a graded $R$-monomorphism and $K$ is a graded $R$-submodule of $f(M)$, then $f^{-1}(K)$ is a graded $R$-submodule of $M$.

2. If $L$ is a graded $R$-submodule of $M$ with $\operatorname{Ker}(f) \subseteq L$, then $f(L)$ is a graded $R$-submodule of $f(M)$.

\section{Proof.}

1. Clearly, $f^{-1}(K)$ is an $R$-submodule of $M$. Let $x \in f^{-1}(K)$. Then $x \in M$ with $f(x) \in K$, and then $x=\sum_{g \in G} x_{g}$ where $x_{g} \in M_{g}$ for all $g \in G$. So, for every $g \in G, f\left(x_{g}\right) \in f\left(M_{g}\right) \subseteq S_{g}$ such that $\sum_{g \in G} f\left(x_{g}\right)=f\left(\sum_{g \in G} x_{g}\right)=f(x) \in K$. Since $K$ is graded, $f\left(x_{g}\right) \in K$ for all $g \in G$, i.e., $x_{g} \in f^{-1}(K)$ for all $g \in G$. Hence, $f^{-1}(K)$ is a graded $R$-submodule of $M$.

2. Clearly, $f(L)$ is an $R$-submodule of $f(M)$. Let $y \in f(L)$. Then $y \in f(M)$, and so there exists $x \in M$ such that $y=f(x)$, so $f(x) \in L$, which implies that $x \in L$ since $\operatorname{Ker}(f) \subseteq L$, and hence $x_{g} \in L$ for all $g \in G$ since $L$ is graded. Thus, $y_{g}=(f(x))_{g}=f\left(x_{g}\right) \in f(L)$ for all $g \in G$. Therefore, $f(L)$ is a graded $R$-submodule of $S$. 
Lemma 3.12. Let $f: M \rightarrow S$ be a graded monomorphism of graded $R$ modules. If $N$ is a graded completely irreducible $R$-submodule of $M$, then $f(N)$ is a graded completely irreducible $R$-submodule of $f(M)$.

Proof. Let $\left\{L_{i}\right\}_{i \in \Delta}$ be a family of a graded $R$-submodules of $f(M)$ such that $f(N)=\bigcap_{i \in \Delta} L_{i}$. Then $N=f^{-1}(f(N))=f^{-1}\left(\bigcap_{i \in \Delta} L_{i}\right)=\bigcap_{i \in \Delta} f^{-1}\left(L_{i}\right)$. So, there exists $i \in \Delta$ such that $N=f^{-1}\left(L_{i}\right)$ since $N$ is a graded completely irreducible $R$-submodule of $M$. Therefore, $f(N)=f\left(f^{-1}\left(L_{i}\right)\right)=$ $f(M) \cap L_{i}=L_{i}$, as needed.

Similarly, one can prove the next lemma.

Lemma 3.13. Let $f: M \rightarrow S$ be a graded monomorphism of graded $R$ modules. If $L$ is a graded completely irreducible $R$-submodule of $f(M)$, then $f^{-1}(L)$ is a graded completely irreducible $R$-submodule of $M$.

Proposition 3.14. Let $f: M \rightarrow S$ be a graded monomorphism of graded $R$-modules. If $N$ is a graded strongly 2-absorbing second $R$-submodule of $M$ with $\operatorname{Ker}(f) \subseteq N$, then $f(N)$ is a graded 2-absorbing second $R$-submodule of $f(M)$.

Proof. Since $N=\{0\}$ and $f$ is injective, we have $f(N)=\{0\}$. Let $x, y \in$ $h(R)$ and $K$ be a graded completely irreducible $R$-submodule of $S$ such that $x y f(N) \subseteq K$. Then $x y N \subseteq f^{-1}(K)$, and then $x N \subseteq f^{-1}(K)$ or $y N \subseteq$ $f^{-1}(K)$ or $x y N=\{0\}$. Therefore, $x f(N) \subseteq f\left(f^{-1}(K)\right)=f(M) \cap K \subseteq K$ or $y f(N) \subseteq K$ or $x y f(N)=\{0\}$, as needed.

Similarly, one can prove the next proposition.

Proposition 3.15. Let $f: M \rightarrow S$ be a graded monomorphism of graded $R$-modules. If $N$ is a graded 2-absorbing second $R$-submodule of $M$ with $\operatorname{Ker}(f) \subseteq N$, then $f(N)$ is a graded 2-absorbing second $R$-submodule of $f(M)$.

Proposition 3.16. Let $f: M \rightarrow S$ be a graded monomorphism of graded $R$-modules. If $L$ is a graded strongly 2 -absorbing second $R$-submodule of $f(M)$, then $f^{-1}(L)$ is a graded 2-absorbing second $R$-submodule of $M$. 
Proof. If $f^{-1}(L)=\{0\}$, then $f(M) \cap L=f\left(f^{-1}(L)\right)=f(\{0\})=\{0\}$. Thus $L=\{0\}$ which is a contradiction. Therefore, $f^{-1}(L)=\{0\}$. Let $x, y \in h(R)$ and $K$ be a graded completely irreducible $R$-submodule of $M$ such that $x y f^{-1}(L) \subseteq K$. Then $x y L \subseteq x y(f(M) \cap L)=x y f\left(f^{-1}(L)\right) \subseteq$ $f(K)$. So, $x L \subseteq f(K)$ or $y L \subseteq f(K)$ or $x y L=\{0\}$. Hence, $x f^{-1}(L) \subseteq K$ or $y f^{-1}(L) \subseteq K$ or $x y f^{-1}(L)=\{0\}$, as needed.

Similarly, one can prove the next proposition.

Proposition 3.17. Let $f: M \rightarrow S$ be a graded monomorphism of graded $R$-modules. If $L$ is a graded 2 -absorbing second $R$-submodule of $f(M)$, then $f^{-1}(L)$ is a graded 2-absorbing second $R$-submodule of $M$.

\section{Graded Quasi 2-Absorbing Second Submodules}

In this section, we introduce and study the concept of graded quasi 2absorbing second submodules.

Definition 4.1. Let $M$ be a graded ring and $N$ be a nonzero graded $R$ submodule of $M$. Then $N$ is said to be a graded quasi 2-absorbing second $R$-submodule of $M$ if $A n n_{R}(N)$ is a graded 2-absorbing ideal of $R$.

Remark 4.2. By Corollary 3.4, every graded strongly 2-absorbing second $R$-submodule is a graded quasi 2-absorbing second $R$-submodule. But the converse is not true in general by Example 3.5.

Proposition 4.3. Let $M$ be a graded comultiplication $R$-module. Then a graded $R$-submodule $N$ of $M$ is a graded strongly 2-absorbing second $R$-submodule of $M$ if and only if it is a graded quasi 2-absorbing second $R$-submodule of $M$.

Proof. It follows from Corollary 3.4 and Proposition 3.7.

Proposition 4.4. Let $M$ be a graded $R$-module and $N$ be a graded quasi 2 -absorbing second $R$-submodule of $M$. If $J$ is a graded ideal of $R$ such that $J \subseteq A n n_{R}(N)$, then $J N$ is a graded quasi 2-absorbing second $R$-submodule of $M$. 
Proof. Since $J \subseteq A n n_{R}(N)$, we have $A n n_{R}(J N)$ is a proper graded ideal of $R$. Let $x, y, z \in h(R)$ such that $x y z \in A n n_{R}(J N)$. Then $x y z J N=$ $\{0\}$, and then $x z N=\{0\}$ or $z y J N=\{0\}$ or $x y J N=\{0\}$. If $z y J N=\{0\}$ or $x y J N=\{0\}$, then we are done. Suppose that $x z N=\{0\}$. Then $x z \in A n n_{R}(N) \subseteq A n n_{R}(J N)$, so $x z J N=\{0\}$, as required.

Proposition 4.5. Let $M$ be a graded $R$-module and $N$ be a graded quasi 2-absorbing second $R$-submodule of $M$. Then $A n n_{R}\left(J^{n} N\right)=A n n_{R}\left(J^{n+1} N\right)$ for all graded ideal $J$ of $R$ and for all $n \geq 2$.

Proof. Let $J$ be a graded ideal of $R$. It is enough to prove that $A n n_{R}\left(J^{2} N\right)=A n n_{R}\left(J^{3} N\right)$. Clearly, $A n n_{R}\left(J^{2} N\right) \subseteq A n n_{R}\left(J^{3} N\right)$. Since $N$ is graded quasi 2-absorbing second, $A n n_{R}\left(J^{3} N\right) J^{3} N=\{0\}$ implies that $A n n_{R}\left(J^{3} N\right) J^{2} N=\{0\}$ or $J^{2} N=\{0\}$. If $A n n_{R}\left(J^{3} N\right) J^{2} N=\{0\}$, then $A n n_{R}\left(J^{3} N\right) \subseteq A n n_{R}\left(J^{2} N\right)$. If $J^{2} N=\{0\}$, then $\operatorname{Ann}_{R}\left(J^{2} N\right)=R=$ $A n n_{R}\left(J^{3} N\right)$.

For a graded $R$-submodule $N$ of $M$, the graded second radical of $N$ is defined as the sum of all graded second $R$-submodules of $M$ contained in $N$, and its denoted by $\operatorname{GSec}(N)$. If $N$ does not contain any graded second $R$-submodule, then $\operatorname{GSec}(N)=\{0\}$. The set of all graded second $R$-submodules of $M$ is called the graded second spectrum of $M$, and is denoted by $G \operatorname{Spec}^{S}(M)$. On the other hand, the set of all graded prime $R$-submodules of $M$ is called the graded spectrum of $M$, and is denoted by $G \operatorname{Spec}(M)$. The map $\phi: G \operatorname{Spec}^{s}(M) \rightarrow G \operatorname{Spec}\left(R / A n n_{R}(M)\right)$ defined by $\phi(N)=\operatorname{Ann}_{R}(N) / A n n_{R}(M)$ is called the natural map of $G \operatorname{Spec}^{s}(M)$, see [9] and [14].

For a graded ideal $I$ of $R$, the graded radical of $I$ is defined to be the set of all $r \in R$ such that for each $g \in G$, there exists a positive integer $n_{g}$ satisfies $r_{g}^{n_{g}} \in I$, and it is denoted by $\operatorname{Grad}(I)$. One can see that if $r \in h(R)$, then $r \in \operatorname{Grad}(I)$ if and only if $r^{n} \in I$ for some positive integer $n$, see $[22]$.

Lemma 4.6. Let $N$ and $K$ be two graded $R$-submodules of $M$. Then

1. $G \operatorname{Sec}(N) \subseteq N$.

2. If $N \subseteq K$, then $G \operatorname{Sec}(N) \subseteq G \operatorname{Sec}(K)$.

3. $\operatorname{GSec}\left(\left(0:_{M} I\right)\right)=\operatorname{GSec}\left(\left(0:_{M} G r(I)\right)\right.$ for all graded ideal $I$ of $R$.

4. $G \operatorname{Sec}(N) \subseteq\left(0:_{M} \operatorname{Gr}\left(A n n_{R}(N)\right)\right)$. 
Proof. (1) and (2) are straightforward. (3) Let $I$ be a graded ideal of $R$. If $G \operatorname{Sec}((0: M I))=\{0\}$, then we are done. Suppose that there exists a graded second $R$-submodule $L$ of $M$ such that $L \subseteq\left(\begin{array}{ll}0: & I\end{array}\right)$. Then $I \subseteq A n n_{R}(L)$. Since $A n n_{R}(L)$ is a graded prime ideal of $R$, we have $\operatorname{Gr}(I) \subseteq A n n_{R}(L)$. Hence, $L \subseteq\left(0:_{M} A n n_{R}(L)\right) \subseteq\left(0:_{M} G r(I)\right)$. Consequently, $G \operatorname{Sec}\left(\left(0:_{M} I\right) \subseteq G \operatorname{Sec}\left(\left(0:_{M} G r(I)\right)\right.\right.$. The reverse inclusion follows by part (2). (4) Since $N \subseteq\left(0:_{M} A n n_{R}(N)\right)$, the result follows by parts (1), (2) and (3).

Lemma 4.7. Let $M$ be a graded $R$-module and $N$ be a graded $R$-submodule of $M$. If the natural map $\phi$ of $G \operatorname{Spec}^{s}(N)$ is surjective, then $\operatorname{Ann}_{R}(G \operatorname{Sec}(N))=$ $\operatorname{Gr}\left(\operatorname{Ann}_{R}(N)\right)$.

Proof. If $N=\{0\}$, then we are done. Suppose that $N=\{0\}$. Then by Lemma 4.6 part (4), we have $G r\left(A n n_{R}(N)\right) \subseteq A n n_{R}(G S e c(N))$. As-

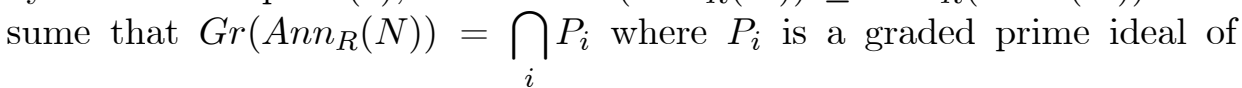
$R$ containing $A n n_{R}(N)$. Since $\phi$ is surjective, for every $P_{i}$, there exists $L_{i} \in \operatorname{GSpec}^{s}(N)$ such that $A n n_{R}\left(L_{i}\right)=P_{i}$. Hence, $\sum_{i} L_{i} \subseteq G \operatorname{Sec}(N)$. Therefore, $A n n_{R}(G \operatorname{Sec}(N)) \subseteq A n n_{R}\left(\sum_{i} L_{i}\right)=\bigcap_{i} P_{i}=G r\left(A n n_{R}(N)\right)$, as needed.

Proposition 4.8. Let $N$ be a graded quasi 2-absorbing second $R$-submodule of $M$. If the natural map $\phi$ of $G \operatorname{Spec}^{s}(N)$ is surjective, then $G \operatorname{Sec}(N)$ is a graded quasi 2-absorbing second $R$-submodule of $M$.

Proof. By Lemma 4.7, $A n n_{R}(G \operatorname{Sec}(N))=\operatorname{Gr}\left(A n n_{R}(N)\right)$, and then the result follows from the fact that $\operatorname{Gr}\left(\operatorname{Ann}_{R}(N)\right)$ is a graded 2-absorbing ideal of $R$ by ([6], Lemma 2.5).

Proposition 4.9. Let $M$ be a graded comultiplication $R$-module, $N \subseteq L$ be two graded $R$-submodules of $M$ and $L$ be a graded quasi 2-absorbing second $R$-submodule of $M$. Then $L / N$ is a graded quasi 2-absorbing second $R$-submodule of $M / N$.

Proof. Let $x, y, z \in h(R)$ such that $x y z(L / N)=\{0\}$. Then $x y z L \subseteq N$, and then $A n n_{R}(N) x y z L=\{0\}$. Thus, $A n n_{R}(N) x y L=\{0\}$ or $A n n_{R}(N) x z L=$ $\{0\}$ or $y z L=\{0\}$. If $y z L=\{0\}$, then $y z(L / N)=\{0\}$, and then we are 
done. If $A n n_{R}(N) x y L=\{0\}$ or $A n n_{R}(N) x z L=\{0\}$, then $x y L \subseteq(0: M$ $\left.A n n_{R}(N)\right)$ or $x z L \subseteq\left(0:_{M} A n n_{R}(N)\right)$. Since $M$ is graded comultiplication, we have $N=\left(0: M A n n_{R}(N)\right)$, and the result follows obviously.

The next example shows that the condition $M$ is a graded comultiplication $R$-module is necessary in Proposition 4.9.

Example 4.10. Let $R=\mathbf{Z}, M=\mathbf{Z}[i]$ and $G=\mathbf{Z}_{2}$. Then $R$ is $G$-graded by $R_{0}=\mathbf{Z}$ and $R_{1}=\{0\}$. Also, $M$ is $G$-graded by $M_{0}=\mathbf{Z}$ and $M_{1}=i \mathbf{Z}$. Clearly, $\mathbf{Z}$ is a graded quasi 2-absorbing second $R$-submodule of $M$. On the other hand, $12 \mathbf{Z}$ is a graded $R$-submodule of $M$ such that $12 \mathbf{Z} \subseteq \mathbf{Z}$ and $\mathbf{Z} / 12 \mathbf{Z}$ is not graded quasi 2-absorbing second. Note that, $M$ is not graded comultiplication $R$-module.

Proposition 4.11. Let $f: M \rightarrow S$ be a graded monomorphism of graded $R$-modules. Then $N$ is a graded quasi 2-absorbing second $R$-submodule of $M$ if and only if $f(N)$ is a graded quasi 2-absorbing second $R$-submodule of $S$.

Proof. It follows from the fact that $A n n_{R}(N)=A n n_{R}(f(N))$.

\section{References}

[1] R. Abu-Dawwas and K. Al-Zoubi, "On graded weakly classical prime submodules", Iranian journal of mathematical sciences and informatics, vol. 12, no. 1, pp. 153-161, 2017, doi: 10.7508/ijmsi.2017.01.012

[2] R. Abu-Dawwas, K. Al-Zoubi and M. Bataineh, "Prime submodules of graded modules", Proyecciones (Antofagasta. On line), vol. 31, no. 4, pp. 355-361, Dec. 2012, doi: 10.4067/ S0716-09172012000400004

[3] R. Abu-Dawwas, M. Bataineh and A. Da'keek, "Graded weak comultiplication modules", Hokkaido mathematical journal, vol. 48, no. 2, pp. 253-261, 2019, doi: 10.14492/ HOKMJ/ 1562810507

[4] K. Al-Zoubi and R. Abu-Dawwas, "On graded quasi-prime submodules", Kyungpook mathematical journal, vol. 55, no. 2 pp. 259-266, 2015, doi: 10.5666/ KMJ.2015.55.2.259

[5] K. Al-Zoubi and R. Abu-Dawwas, "On graded 2-absorbing and weakly graded 2-absorbing submodules", Journal of mathematical sciences: advances and applications, vol. 28, pp. 45-60, 2014. [On line]. Available: https:/ / bit.ly/ 34KEQ1x 
[6] K. Al-Zoubi, R. Abu-Dawwas, and S. Ceken, "On graded 2-absorbing and graded weakly 2-absorbing ideals", Hacettepe journal of mathematics and statistics, vol. 48, no. 3, pp. 724-731, 2019, doi: 10.15672/ hjms.2018.543

[7] K. Al-Zoubi and M. Al-Azaizeh, "Some properties of graded 2-absorbing and graded weakly 2-absorbing submodules", Journal of nonlinear sciences and applications, vol. 12, no. 8, pp. 503-508, Mar. 2019, doi: 10.22436/jnsa.012.08.01

[8] K. Al-Zoubi, M. Jaradat and R. Abu-Dawwas, "On graded classical prime and graded prime submodules", Bulletin of the iranian mathematical society, vol. 41, no. 1, pp. 205-2013, 2015. [On line]. Available: https:/ / bit.ly/ 383ka60

[9] H. Ansari-Toroghy and F. Farshadifar, "On graded second modules", Tamkang journal of mathematics, vol. 43, no. 4, pp. 499-505, 2012, doi: 10.5556/ j.tkjm.43.2012.1319

[10] H. Ansari-Toroghy and F. Farshadifar, "Graded comultiplication modules", Chiang mai journal of science, vol. 38, no. 3, pp. 311-320, Jul. 2011. [On line]. Available: https:/ / bit.ly/ 340e48w

[11] H. Ansari-Toroghy and F. Farshadifar, "Some generalizations of second submodules", Palestine journal of mathematics, vol. 8, no. 2, pp. 159168, 2019. [On line]. Available: https:/ / bit.ly/ 2JmQKGu

[12] S. E. Atani, "On graded prime submodules", Chiang mai journal of science, vol. 33, no. 1, pp. 3-7, 2006. [On line]. Available: https:/ / bit.ly/ 2Jz1nGB

[13] S. E. Atani and F. Farzalipour, "On graded secondary modules", Turkish journal of mathematics, vol. 31, no. 4, 2007, pp. 371-378. [On line], Available: https:/ / bit.ly/ 3 jMSnd3

[14] S. Çeken and M. Alkan, "On graded second and coprimary modules and graded second representations", Bulletin of Malaysian Mathematical Society, vol. 38, no. 4, Oct. 2015, pp. 1317-1330, doi: 10.1007/ s40840-014-0097-6

[15] J. Chen and Y. Kim, "Graded irreducible modules are irreducible", Communications in algebra, vol. 45, no. 5, pp. 1907-1913, Oct. 2017, doi: 10.1080/ 00927872.2016.1226864

[16] F. Farzalipour and P. Ghiasvand, "On the union of graded prime submodules", Thai journal of mathematics, vol. 9, no. 1, pp. 49-55, 2011. [On line]. Available: https:/ / bit.ly/ 38423xD

[17] F. Farzalipour and P. Ghiasvand, "On graded weak multiplication modules", Tamkang journal of mathematics, vol. 43, no. 2, pp. 171-177, 2012, doi: $10.5556 /$ j.tkjm.43.2012.712 
[18] L. Fuchs, W. Heinzer, and B. Olberding, "Commutative ideal theory without finiteness conditions: irreducibility in the quotient field," in Abelian groups, rings, modules, and homological algebra, P. Goeters and O. M. G. Jenda , Eds. Boca Raton, CA: Chapman and Hall/ CRC, 2006, pp. 121-145.

[19] C. Meng, "G-graded irreducibility and the index of reducibility", Communications in algebra, vol. 48, no. 2, pp. 826-832, Oct. 2019. doi: 10.1080/ 00927872.2019.1662914

[20] S. R. Naghani and H. F. Moghimi, "On graded 2-absorbing and graded weakly 2-absorbing ideals of a commutative ring", Çankaya University journal of science and engineering, vol. 13, no. 2, pp. 11-17, 2016, doi: 10.15672/ hjms.2018.543

[21] C. Năstăsescu and F. V. Oystaeyen, Methods of graded rings. Berlin: Springer, 2004, doi: 10.1007/ b94904

[22] M. Refai and K. Al-Zoubi, "On graded primary ideals", Turkish journal of mathematics, vol. 28, no. 3, pp. 217-229, 2004. [On line]. Available: https:/ / bit.ly/ 34QIO91

[23] J. Zhang, "A 'natural' graded Hopf algebra and its graded Hopf-cyclic cohomology", Journal of noncommutative geometry, vol. 6, no. 2, pp. 389- 405, 2012. [On line]. Available: https:/ / bit.ly/ 3jQsTLW 